\title{
Ökologische Untersuchungen an Modellfliessgewässern
}

\begin{abstract}
III. Die jahreszeitlichen Veränderungen im Verhältnis von heterotropher zu phototropher Biomasse bei verschiedenen Abwasserbelastungen
\end{abstract}

\author{
Von E. EICHENBERGER
}

Eidgenössische Anstalt für Wasserversorgung, Abwasserreinigung und Gewässerschutz.

(EAWAG) an der ETHZ, Dübendorf

\begin{abstract}
The seasonal changes of the biomass production by benthic microphytes have been investigated in outdoor channels (breadth $25 \mathrm{~cm}$, depth $10-20 \mathrm{~cm}$, flow $41 / \mathrm{sec}$ ) for the first 20 days of colonisation. The channels were fed with groundwater to which $0,1,5$ and $12 \%$ settled sewage was added as pollutant. The heterotrophic and the phototrophic portions of the total growth were estimated on the basis of the chlorophyll content of the total biomass. Addition of $12 \%$ sewage caused an increase of the heterotrophic biomass at a constant rate during the first 3 to 4 weeks of colonisation. For each season tested the heterotrophic growth is proportional to the sewage concentration within the range from 0 to $12 \%$. At any given sewage concentration the ratio between phototrophic and heterotrophic microphytes undergoes a seasonal variation. Best phototrophic growth occurs in summer in 1 to $5 \%$ sewage. Heterotrophic microphytes find better conditions in winter. With an addition of $5 \%$ sewage the proportion of phototrophic microphytes in the total biomass is about $10 \%$ in winter, and around $70 \%$ in summer. At a sewage concentration of $12 \%$ the proportion ranges between $2 \%$ and $30 \%$. Light incidence represents an important variable for the growth of heterotrophic microphytes.
\end{abstract}

\section{Einleitung}

Die an natürlichen Gewässern festgestellte Förderung des benthischen Wachstums durch die Einleitung von häuslichem Abwasser lässt sich auch in Freilandmodellen beobachten [15]. Neben den heterotrophen Mikrophyten, die über einen breiten Belastungsbereich gefördert werden, sprechen auch eine grosse Zahl von benthischen Algen auf eine mässige Abwasserzufuhr mit kräftigem Wachstum an. Die klimatischen Ansprüche der einzelnen Arten weichen zum Teil erheblich voneinander ab und können durch die Abwasserkonzentration modifiziert werden. Daraus resultieren bei gleichbleibender Abwasserverdünnung jahreszeitliche Veränderungen im mengenmässigen Anteil der phototrophen und heterotrophen Komponenten an der Biomasse [7].

Unter den vielen Parametern, die für die Beurteilung des Geschehens in einem verunreinigten Gewässer von Bedeutung sind, stehen die Biomasse des benthischen 
Bewuchses und ihre Verteilung auf phototrophe und heterotrophe Mikrophyten im Vordergrund. Erst die Kenntnis dieser Grössen und ihrer Zuwachsgeschwindigkeit erlaubt die Interpretation von Beobachtungen über den Ablauf physiologischer Prozesse, wie z.B. des Gashaushalts und der Selbstreinigung [18, 19].

Die Bestimmung der Zuwachsgeschwindigkeit des mikrophytischen Benthos bietet Schwierigkeiten verschiedener Art, die zum Teil durch die kurze Lebensdauer und die Hinfälligkeit der Organismen des Bewuchses bedingt sind. Die Möglichkeit der quantitativen Untersuchung der Auswirkung einer Abwassereinleitung auf das Wachstum von benthischen Mischbiozönosen wird dadurch eingeschränkt und ist an gewisse Voraussetzungen gebunden. Die vorliegende Arbeit soll abklären, in welcher Phase der Besiedlung die Zuwachsgeschwindigkeit des Benthos zuverlässig gemessen werden kann, und in welcher Weise die Biomassebildung der phototrophen und heterotrophen Mikrophyten zu verschiedenen Jahreszeiten von der Abwasserbelastung abhängt.

\section{Versuchseinrichtungen und Methodik}

\subsection{Die Versuchsanlage}

Die Versuchsanlage besteht aus im Freien aufgestellten, etwa $200 \mathrm{~m}$ langen Betonkanälen von trapezförmigem Querschnitt (Basisbreite $25 \mathrm{~cm}$, Kronenbreite $25 \mathrm{~cm}$, Tiefe $20 \mathrm{~cm}$ ) mit einem gleichmässigen Gefälle von 1,5\% (weitere Angaben siehe $[17,6])$.

\subsection{Quantitative Bestimmung der Biomasse}

Als Biomasse bezeichnen wir hier das gesamte Material, das an einer Stelle abgeerntet werden kann. Da alle Versuche kurzfristig laufen, ist der Anteil von zerfallenen Organismen und Schlamm an der Biomasse in der Regel gering.

Die Biomassebestimmung erfolgt in derselben Weise wie in früheren Arbeiten [7], so dass hier nur die wichtigsten Angaben folgen:

Bewuchsernte: Bei abgestellter Wasserzufuhr wird der gesamte Bewuchs von $1 / 2 \mathrm{~m}$ langen Rinnenabschnitten quantitativ abgeerntet (etwa $1 / 4 \mathrm{~m}^{2}$ Bewuchsfläche).

Frischgewicht: Das gesamte Erntegut wird für 5 Min. bei etwa $1000 \mathrm{~g}$ abzentrifugiert. Trockengewicht: Das gesamte Erntegut wird bei $105^{\circ} \mathrm{C}$ für 2 Tage in einem Ventilatortrockenschrank mit Lufterneuerung getrocknet.

Glühverlust: Gut durchgemischte Proben des Trockenguts werden bei $650^{\circ} \mathrm{C}$ für 4 Stunden geglüht.

Chlorophyllgehalt: Proben von $1 \mathrm{~g}$ Frischgewicht werden mit $50 \mathrm{ml}$ heissem Methanol extrahiert. Die Extinktion ( $2 \mathrm{~cm}$ Weglänge) des entsprechend verdünnten Extrakts wird bei $663 \mathrm{~m} \mu$ in einem Leitz-Elko-II-Kolorimeter bestimmt. Die auf $50 \mathrm{ml}$ Methanol umgerechneten fiktiven Extinktionswerte werden als relativer Chlorophyllgehalt/g Frischgewicht bezeichnet. Mit dem Frischgewicht pro Einheitsfläche multipliziert ergibt sich der relative Chlorophyllgehalt pro Einheitsfläche.

$P / H$-Index: Wir bezeichnen das Verhältnis von relativem Chlorophyllgehalt zu Glühverlust einer Probe als $\mathrm{P} / \mathrm{H}$-Index. $\mathrm{P} / \mathrm{H}$-Indizes des Rinnenbewuchses bewegen sich zwischen 1 und etwa 50, wobei hohe Werte charakteristisch sind für phototrophenreiche Biozönosen, tiefe für heterotrophenreiche.

Scheidung zwischen phototropher und heterotropher Biomasse: Da eine mechanische Trennung der phototrophen von den heterotrophen Mikrophyten nicht in Frage 
kommt, erfolgt die Scheidung auf Grund des Chlorophyllgehalts des Bewuchses. Der Chlorophyllgehalt der Algen ist artlichen und klimatischen Variationen unterworfen [14], so dass mit variablen $\mathrm{P} / \mathrm{H}$-Indizes für Algen verschiedener Standorte zu rechnen ist. Erfahrungsgemäss liegt in unseren Rinnen für einen reinen Algenbewuchs im Alter von 20 Tagen der P/H-Index bei einem Wert von etwa 40 (Extinktionseinheiten/g Glühverlust). Dieser Wert wurde als Basis für die Scheidung in phototrophe und heterotrophe Biomasse eingesetzt.

Zur Bestimmung der Teilbiomasse wird folgendermassen verfahren:

Phototrophe Biomasse $=$ Chlorophyllgehalt der Gesamtbiomasse dividiert durch $\mathrm{P} / \mathrm{H}$-Index (40), heterotrophe Biomasse = Gesamtbiomasse minus phototrophe Biomasse.

Als Einheitsfläche gilt die Bewuchsfläche, d. h. jener Teil des benetzten Profils, der vom Bewuchs eingenommen wird.

\subsection{Betriebsbedingungen}

Abwasser: Das den Rinnen zugeleitete Abwasser ist mechanisch vorgeklärtes häusliches Abwasser aus dem Hauptabwasserkanal der Stadt Zürich.

Wasserchemismus: Die Bedingungen sind in einer früheren Arbeit beschrieben worden [6] und gelten auch für diese Versuche, die in den Jahren 1967 und 1968 durchgeführt wurden.

Durchfluss: Alle Versuche erfolgen bei einem Durchfluss von $41 / \mathrm{sec}$.

Rinnensohle: Die Rinnensohle besteht aus rauhem Beton und ist im Gegensatz zu früheren Versuchen nicht mit Kies ausgelegt.

Fliessgeschwinaigkeit: In den unbesiedelten Kanälen beträgt sie bei einem Durchfluss von 4 1/sec etwa $30 \mathrm{~cm} / \mathrm{sec}$. Je nach dem Grad der Ausbildung des Bewuchses liegt die Fliessgeschwindigkeit in den besiedelten Kanälen zwischen 15 und $25 \mathrm{~cm} / \mathrm{sec}$. Als Fliessgeschwindigkeit gilt die mittlere Fortpflanzungsgeschwindigkeit des Maximums eines Salzstosses.

Vorbehandlung der Gerinne: Vor dem Ansatz der Experimente wurde der gesamte Bewuchs mit einer rotierenden Stahlbürste entfernt.

Beimpfung: Sie erfolgt durch Keime, die aus der Luft oder im Abwasser eingeführt werden.

\subsection{Die Versuchsanordnung}

Die Entwicklung der Biozönosen in den Rinnen durchläuft drei mehr oder weniger deutlich voneinander geschiedene Stadien [9]:

1. Besiedlungsphase: der freie Wohnraum wird kolonisiert und bewachsen. Die Biomasse nimmt stetig zu.

2. Biotopsättigung ("saturation population» nach Phaup und Gannon [12]): Abtrieb, Frass durch Tiere und Neubildung stehen angenähert in einem Gleichgewicht. Die stehende Biomasse verändert sich nur wenig.

3. Zerfall der Primärbiozönose: Je nach der Art der Besiedlung erfolgen massive Ablösungen des Bewuchses, d. h. der Abtrieb übertrifft die Neubildung.

Bei konstanter Wasserzufuhr und einheitlichem Profil der Gerinne steigt das Wasserniveau als Folge der Volumenzunahme des Bewuchses und des Widerstandes, den er dem strömenden Wasser entgegensetzt. Sofern keine Veränderungen in der Bewuchsstruktur erfolgen, wie z. B. ein Übergang von einem krustenartigen in einen 
flutenden Bewuchs, korrelieren Wasserniveau und Biomasse gut. Pegelstandsmessungen erlauben unter diesen Umständen die kontinuierliche Beobachtung der Bewuchsentwicklung ohne Störung der Biozönose.

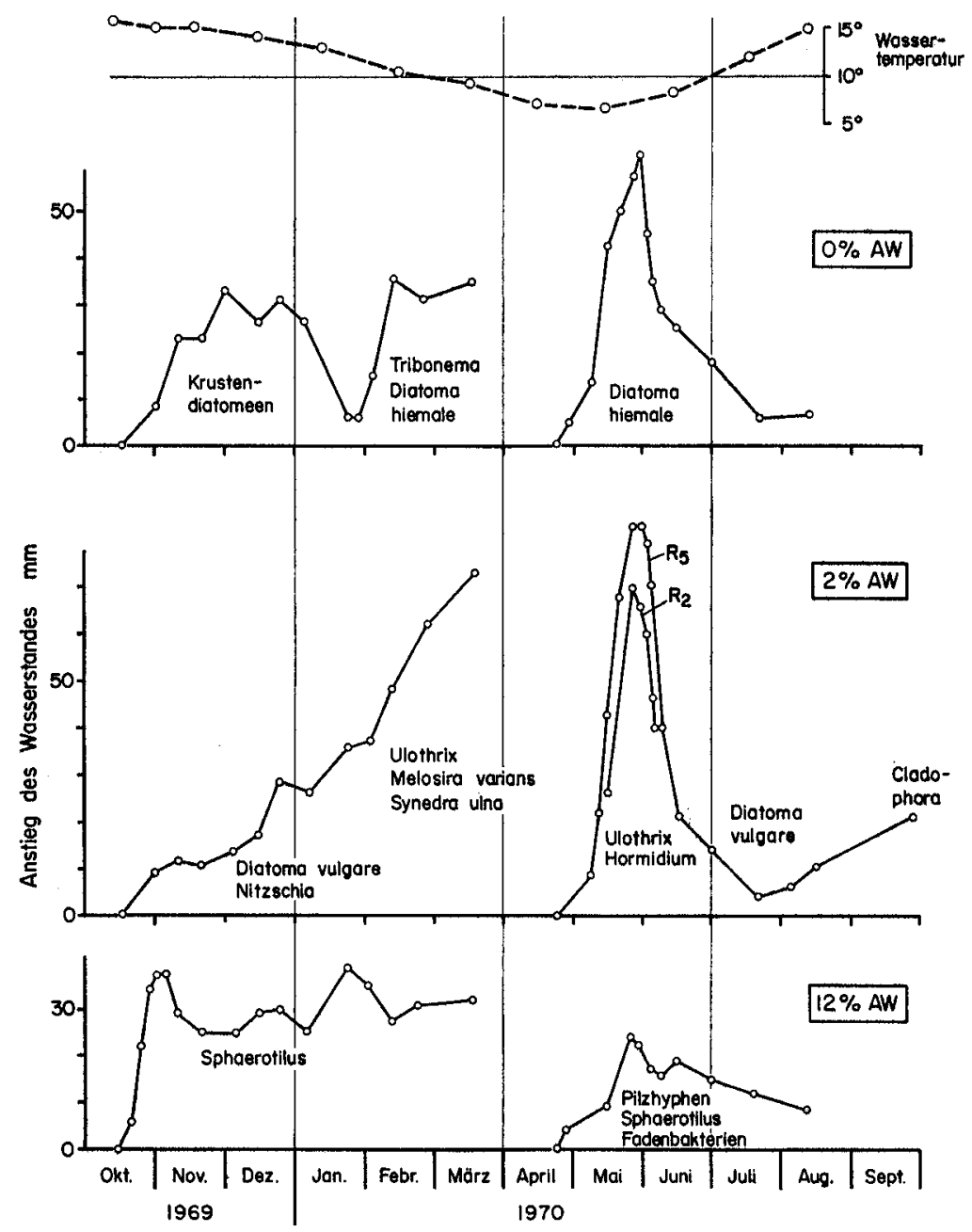

$\mathrm{Abb} .1$. Veränderung der Bewuchsdichte in ungestörten Biozönosen bei 0,2 und $12 \%$ Abwasserzugabe, gemessen an den Veränderungen des Wasserstands in den Rinnen.

Messungen am Anfang der Rinnen. Angabe der dominierenden Organismen der Biozönosen. Ende März 1970 wurde der gesamte Bewuchs entfernt. $R_{5}$ und $R_{2}$ in $2 \%$ Abwasser: Vergleich von 2 Rinnen, die unter denselben Versuchsbedingungen stehen.

Fig. 1. Changes in the density of the vegetation in channels with 0,2 and $12 \%$ sewage added (recorded as change in the water level). $R_{2}$ and $R_{5}$ with $2 \%$ sewage: comparison of 2 channels under the same experimental conditions.

The organisms most characteristic for the biocenosis are inserted. At the end of March 1970 the vegetation was completely removed. 
Wie aus Abb. 1 hervorgeht, fällt die Dauer der Besiedlungsphase je nach den Bedingungen und der Art der Besiedlung sehr unterschiedlich aus und bewegt sich zwischen einem und mehreren Monaten. Da während der Besiedlungsphase die Verhältnisse übersichtlicher erscheinen, z. B. wenig Biomasse durch Abtrieb verlorengeht oder biotische Faktoren, wie etwa Prädation oder Alterungsprozesse, noch von untergeordneter Bedeutung sind, wurde die Biomasseproduktion nur in der Besiedlungsphase untersucht.

\section{Versuchsergebnisse}

\subsection{Der zeitliche Verlauf der Biomassebildung in der Besiedlungsphase}

\subsection{Heterotrophe Biozönosen (12\% Abwasser)}

Der zeitliche Verlauf der Biomassebildung lässt sich durch sigmoide Kurven darstellen, die in den ersten 3-4 Wochen annähernd mit einer Geraden zusammenfallen (Abb. 2). Die Korrelationskoeffizienten zwischen Biomasse und Besiedlungsdauer liegen für diesen Zeitabschnitt am Rinnenanfang um 0,98. In unterliegenden Probeabschnitten erfolgt die partielle Ablösung des Bewuchses oft etwas früher, so dass hier mit grösseren Streuungen zu rechnen ist. Dieser Prozess steht im Zusammenhang mit der fortschreitenden Selbstreinigung längs der Fließstrecke [8]. Die in Abb. 2 wiedergegebenen Daten wurden an 3 gleichzeitig betriebenen Rinnen gewonnen und zeigen die gute Reproduzierbarkeit der Bewuchsbildung unter gleichen Versuchsbedingungen. Auch in komplexer aufgebauten Mischbiozönosen bei geringerer Ab-

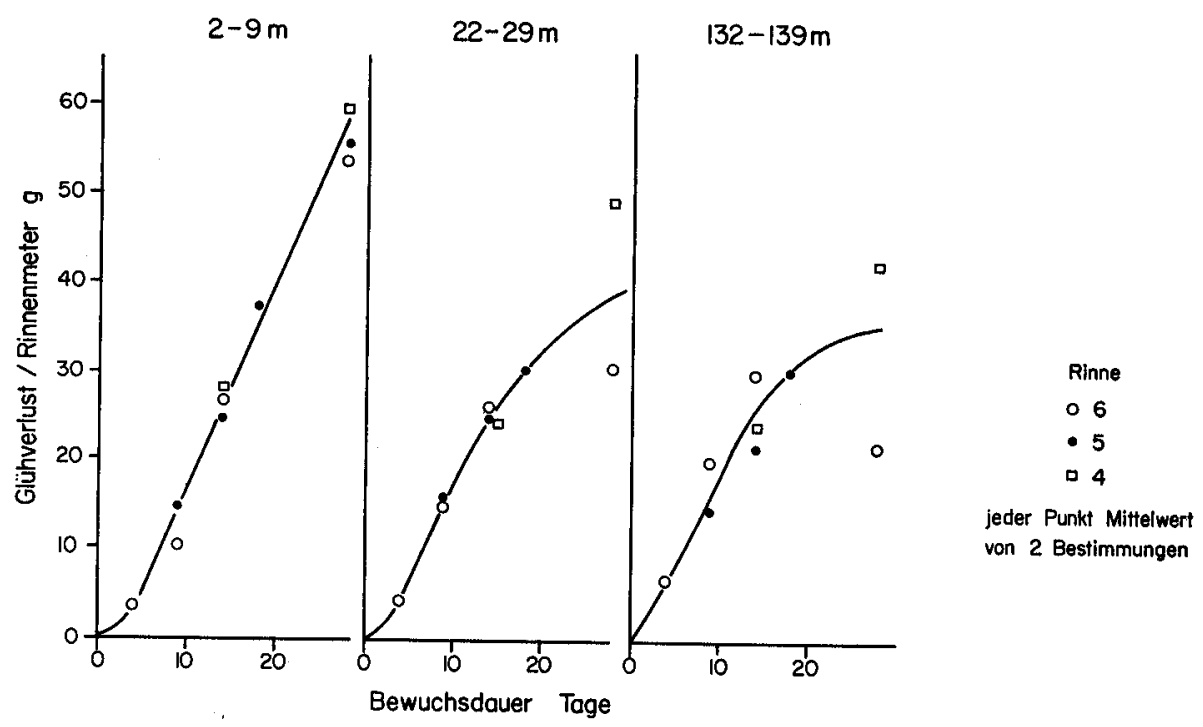

Abb. 2. Zcitlicher Verlauf der heterotrophen Biomassebildung in 3 mit 12\% Abwasser beschickten Rinnen nach 3 verschiedenen Fliessdistanzen.

Fig. 2. Accumulation of the heterotrophic biomass in 3 channels with $12 \%$ sewage as a function of time and flow distance.

Each point represents the average of 2 biomass determinations. Biomass: $g$ organic substance (i.e. ignition loss)/meter channel. 
wasserbelastung kann eine gute Übereinstimmung der Biomasseentwicklung und der Verteilung auf den phototrophen und heterotrophen Anteil festgestellt werden (Abb. $3 ; 2 \%$ Abwasser).

Aus den hier angeführten Werten und weiteren damit übereinstimmenden Ergebnissen anderer Versuche ergibt sich, dass während der ersten 20 Besiedlungstage in einer heterotrophen Biozönose die Biomasse proportional zur Zeit ansteigt.

\subsection{Phototrophe Biozönosen}

Phototrophe Biozönosen weisen grössere Unterschiede in ihrer Struktur auf als heterotrophe. Zwischen den Extremen einer kompakten epilithischen Kruste und einer gleichmässigen räumlichen Verteilung von flutenden Algenfäden oder flutenden Diatomeenzotten sind viele Übergänge möglich. Doch erfolgt in der Regel auch bei phototrophen Biozönosen der Biomassezuwachs während der Besiedlungsphase angenähert proportional zur Zeit (Abb. 1; [7], Abb. 3).

\subsection{Bestimmung der Wachstumsgeschwindigkeit der Biozönosen}

Die Wachstumsgeschwindigkeiten wurden durchwegs aus Versuchen mit einer maximalen Besiedlungsdauer von 21 Tagen gewonnen. Aus der stehenden Biomasse bei Versuchsende wurde die mittlere Zuwachsgeschwindigkeit berechnet und in $\mathrm{g}$ Glühverlust/Tag $\cdot \mathrm{m}^{2}$ Bewuchsfläche ausgedrückt (für $\mathrm{g} \mathrm{C} / \mathrm{Tag} \cdot \mathrm{m}^{2}$ Bewuchsfläche mit etwa $0,5 \mathrm{zu}$ multiplizieren).

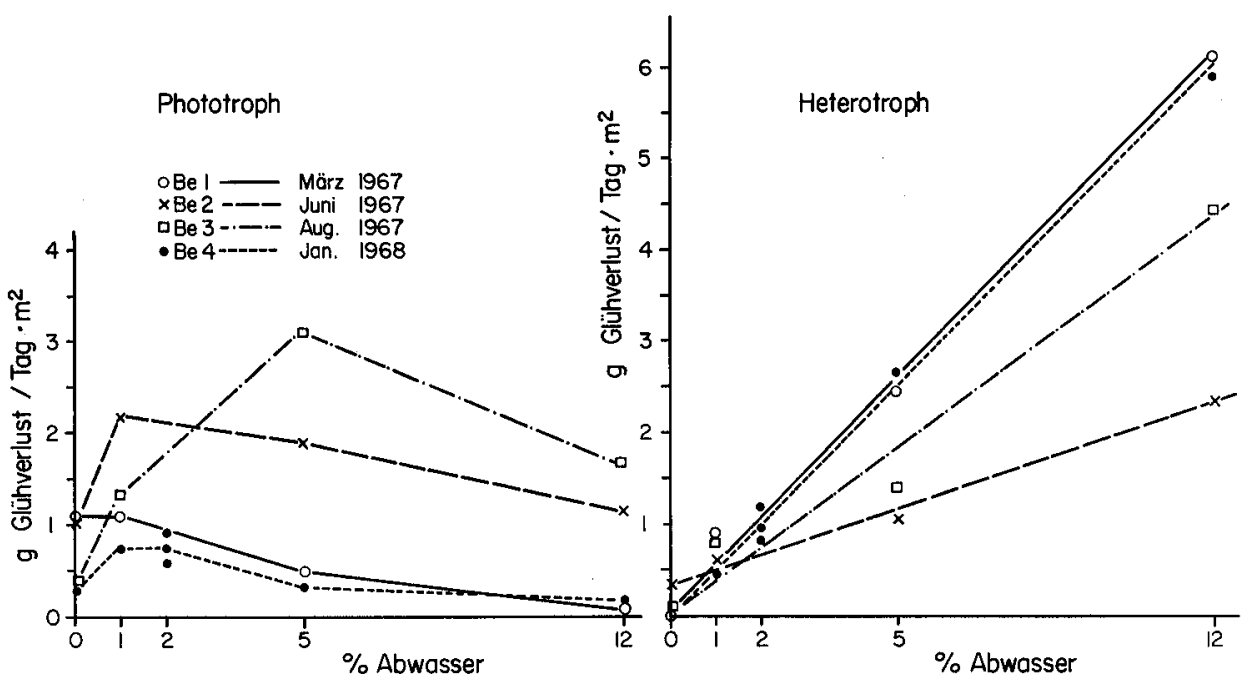

$\mathrm{Abb}$. 3. Entwicklung der heterotrophen und der phototrophen Biomasse bei verschiedenen $\mathrm{Ab}-$ wasserdosierungen zu verschiedenen Jahreszeiten. Besiedlungsdauer: je 20 Tage.

Fig. 3. Development of the phototrophic and the heterotrophic biomass as a function of the sewage load and the season.

Growth rate: $g$ organic substance (i. e. ignition loss)/day and $\mathrm{m}^{2}$ overgrown surface. Duration of colonisation: 20 days. 
Tabelle 1. Die wichtigsten bestandesbildenden Organismen nach 20 Tagen Besiedlungsdauer.

\begin{tabular}{|c|c|c|c|c|c|c|c|c|c|c|c|c|c|c|c|c|}
\hline \multirow[t]{2}{*}{ Abwasser } & \multicolumn{4}{|l|}{$0 \%$} & \multicolumn{4}{|l|}{$1 \%$} & \multicolumn{4}{|l|}{$5 \%$} & \multicolumn{4}{|l|}{$12 \%$} \\
\hline & Jan. & März & Juli & Sept. & Jan. & März & Juli & Sept. & Jan. & März & Juli & Sept. & Jan. & März & Juli & Sept. \\
\hline Heterotrophe Biomasse $\mathrm{g} / \mathrm{Tag} \cdot \mathrm{m}^{2}$ & 0,00 & 0,00 & 0,00 & 0,06 & 0,43 & 0,89 & 0,58 & 0,86 & 2,64 & 2,43 & 1,06 & 1,40 & 6,20 & 6,10 & 2,34 & 4,45 \\
\hline Heterotrophe & - & - & - & - & $\mathbf{1}-2$ & 1 & 1 & 1 & $2-3$ & $2-3$ & 2 & 2 & 4 & 4 & $2-3$ & 3 \\
\hline Phototrophe Biomasse $\mathrm{g} / \mathrm{Tag} \cdot \mathrm{m}^{2}$ & 0,30 & 1,07 & 1,06 & 0,36 & 0,74 & 1,09 & 2,18 & 1,32 & 0,36 & 0,46 & 1,95 & 3,10 & 0,12 & 0,13 & 1,12 & 1,68 \\
\hline Fadenalgen & - & 2 & 1 & + & $1-2$ & $2-3$ & 2 & $1-2$ & - & $1-2$ & $1-2$ & 2 & - & - & 1 & - \\
\hline Ulothrichaceen & - & - & - & - & $1-2$ & 2 & 2 & - & - & 1 & $1-2$ & - & - & - & 1 & - \\
\hline Tribonema & - & 2 & 1 & + & - & 2 & 1 & $1-2$ & - & 1 & 1 & 2 & - & - & - & - \\
\hline Diatomeen & $1-2$ & $2-3$ & 2 & 1 & $1-2$ & $2-3$ & 3 & 2 & + & $1-2$ & $2-3$ & $2-3$ & - & - & $1-2$ & - \\
\hline Meridion circulare & - & $2-3$ & - & - & - & - & - & - & - & - & - & - & - & - & - & - \\
\hline Diatoma vulgare & - & - & - & + & - & - & 3 & - & - & - & $1-2$ & & - & - & - & - \\
\hline Synedra ulna & - & - & - & - & - & 2 & - & - & - & - & 2 & $2-3$ & - & - & - & - \\
\hline $\begin{array}{l}\text { Krustendiatomeen } \\
\text { (Achnanthes ssp.) }\end{array}$ & $1-2$ & 2 & 2 & + & $1-2$ & + & $1-2$ & 2 & $1-2$ & - & - & - & - & - & - & - \\
\hline $\begin{array}{l}\text { Nitzschia ssp. (vorwiegend } \\
N . \text { fonticola und palea) }\end{array}$ & - & - & - & - & 1 & 2 & - & - & + & 2 & 2 & 2 & - & - & $1-2$ & $1-2$ \\
\hline Phormidiumlager & + & + & - & - & + & - & - & - & - & - & - & + & - & - & - & - \\
\hline
\end{tabular}

Skala für die Schätzung der Beruchsdichte

4 Sehr dicht: Völlige Kolonisation des zur Verfügung stehenden Siedlungsraums.

3 Dicht: Aber der verfügbare Raum wird nicht voll besiedelt.

2 Locker: Der betreffende Organismus fällt durch stellenweise kräftige Entwicklung auf, bildet aber keine gleichmässig deckenden Bestänđe, oder aber er ist gleichmässig, aber in geringer Dichte verteilt.

1 Vereinzelt: Ist in kleinen, lokalen Agglomerationen oder gleichmässig dünn verteilt vorhanden, bildet aber keine grösseren Bestände und fällt daher wenig auf.

$+\quad$ orhanden: Kann bei sorgfältiger Prüfung noch von blossem Auge festgestellt werden. 


\subsection{Der Einfluss der Abwasserkonzentration auf die Biomassebildung}

Die Zuwachsgeschwindigkeit der Heterotrophen steigt bis zu einer Beigabe von $12 \%$ Abwasser angenähert proportional zur Abwasserlast an (Abb. 3). Diese lineare Zunahme erfolgt sowohl bei guten als auch bei schlechten Wachstumsbedingungen.

Das Entwicklungsoptimum für Algen verteilt sich auf einen breiten Bereich von Abwasserbelastungen (Tab. 1, Abb. 3). Für die Mehrzahl der Arten liegt es zwischen 0 und $5 \%$; einige Arten bevorzugen aber höhere Konzentrationen (einige kokkoide Grünalgen, Nitzschia palea). Daraus resultieren die diskontinuierliche Verteilung der Arten im Abwassergradienten und die oft sehr ausgeprägten Unterschiede in der Struktur des phototrophen Bewuchses.

\subsection{Der jahreszeitliche Verlauf der Biomassebildung}

Das augenfälligste Merkmal in der jahreszeitlichen Verteilung der Mikrophyten ist die Zunahme der Phototrophen und der Rückgang der Heterotrophen im Sommerhalbjahr. Nach unseren bisherigen Erfahrungen erfolgt die Entwicklung der schnellwachsenden Phototrophen in den Modellfliessgewässern in 3 Schüben $[6,7]$ :

1. Im Frühjahr (März bis Mai, Wassertemperatur $6-10^{\circ} \mathrm{C}$ ): die Ulothrichaceen;

2. Im Frühsommer (Mai bis Juni, Wassertemperatur $6-10^{\circ} \mathrm{C}$ ): zottenbildende Diatomeen (vor allem Diatoma vulgare);

3. Im Spätsommer (August bis September, Wassertemperatur $13-18^{\circ} \mathrm{C}$ ): Cladophora sp.

Die Versuche wurden in die voraussichtliche Hauptentwicklungszeit dieser wichtigsten bestandesbildenden Phototrophen gelegt. Aus Umständen, die vorderhand noch nicht abgeklärt sind, sich zum Teil aber auf veränderte Beimpfungsbedingungen zurückführen lassen, war das Algenwachstum im Versuchsjahr 1967 ausgesprochen schlecht. Die Reaktion einzelner Algengruppen auf steigende Abwasserbelastung konnte daher nicht in der geplanten Weise untersucht werden. Trotz dem Ausbleiben der in früheren Jahren üblichen Massenentwicklungen von Ulothrix im Frühjahr und von Cladophora im Frühherbst weist der jahreszeitliche Wechsel des Verhältnisses zwischen Phototrophen und Heterotrophen (Abb. 4) eine gute generelle Übereinstimmung mit früheren Beobachtungen $[6,7]$ auf.

Bei einer geringen Abwasserkonzentration von $1 \%$ bewegt sich die Heterotrophenproduktion im Laufe des Jahres zwischen 0,5 und etwa $1 \mathrm{~g}$ Glühverlust/Tag $\cdot \mathrm{m}^{2}$, diejenige der Phototrophen zwischen 0,5 und 2. Bei höheren Abwassergehalten sind diese Schwankungen der beiden Komponenten der Biomasse noch ausgeprägter. Der Anteil der Phototrophen an der Gesamtbiomasse variiert bei $5 \%$ Abwasserzugabe zwischen etwa 10 und $70 \%$, bei $12 \%$ Abwasserzugabe zwischen 2 und $30 \%$.

Der heterotrophe Bewuchs in 12\% Abwasser zeigt im Laufe des Jahres eine deutliche Veränderung seiner Zusammensetzung. In den Biozönosen werden zwei verschiedene Wuchsformen festgestellt:

1. Ein heller Bewuchs mit lockeren Zotten, der vorwiegend aus Sphaerotilus natans aufgebaut ist.

2. Ein kompakter, mehr dunkelgrauer, pelziger Bewuchs, mit mehr oder weniger glatter Oberfläche, ohne flutende Zotten. Er weist einen hohen Anteil von Pilzhyphen 


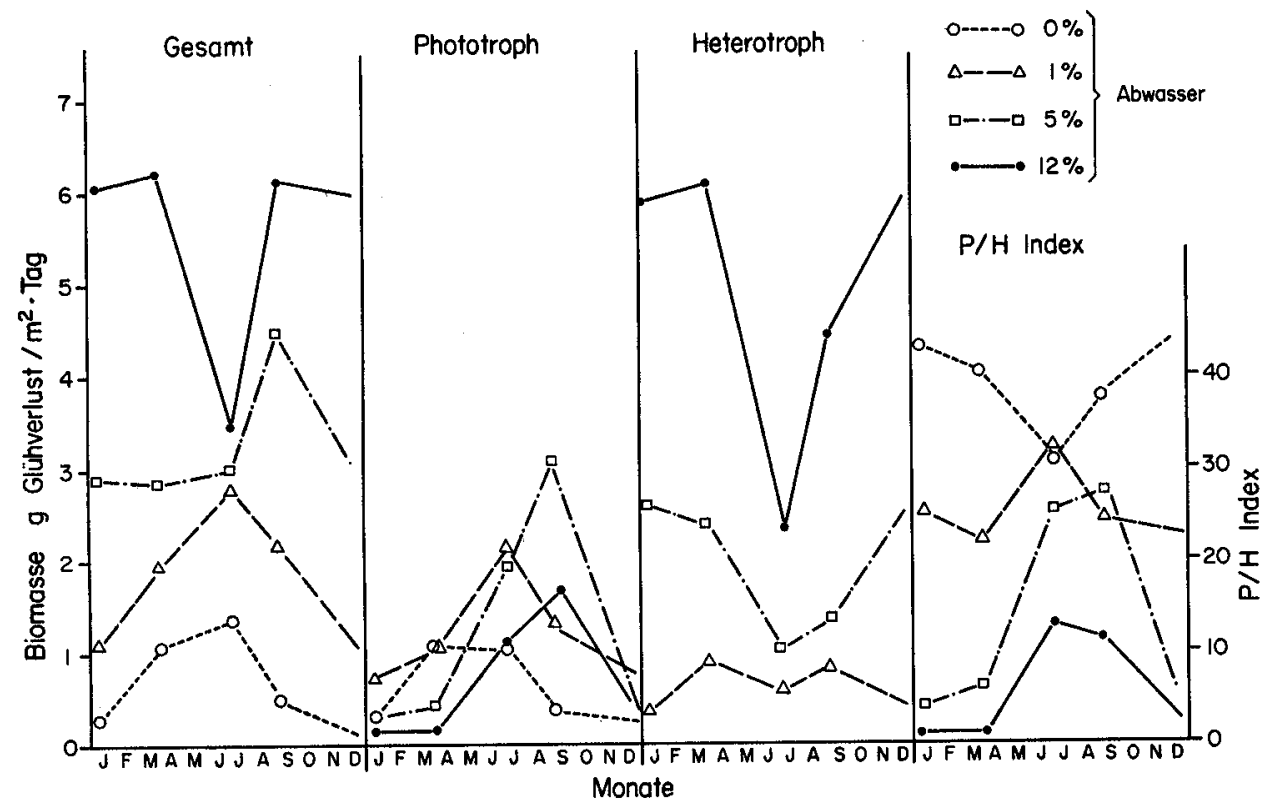

Abb. 4. Die jahreszeitliche Verteilung der Zuwachsgeschwindigkeit des phototrophen und des heterotrophen Anteils der Biozönosen bei verschiedenen Abwasserbelastungen. Besiedlungsdauer: je 20 Tage.

Fig. 4. Seasonal variation of the phototrophic and the heterotrophic growth rates of the biocenosis grown in 4 different sewage loads.

Growth rate: $g$ organic substance (i.e. ignition loss)/day and $\mathrm{m}^{2}$ overgrown surface. Duration of colonisation : 20 days.

(wahrscheinlich den Fungi imperfecti angehörend) und feinen Fadenbakterien auf (wahrscheinlich Flavobakterien). Bei typischer Ausbildung dieser Wuchsform macht Sphaerotilus wahrscheinlich weniger als die Hälfte der Biomasse aus.

Die beiden Wuchsformen können bei hoher Abwasserbelastung gelegentlich in der gleichen Rinne ein unregelmässiges Mosaik bilden; doch hat in der Regel in den letzten Jahren bei tieferen Wassertemperaturen der pilzreiche, bei höheren der sphaerotilusreiche Bewuchs vorgeherrscht. Die beiden Ausbildungen der heterotrophen Biozönosen unterscheiden sich deutlich in ihrem Wasserhaltevermögen. Für reinen Sphatrotilusbewuchs liegt der Anteil der Trockensubstanz am Frischgewicht unter $5 \%$, bei einer typischen pelzigen Ausbildung mit viel Pilzhyphen und Bakterienlagern über 16\%. Der Anteil der Trockensubstanz am Frischgewicht wird daher zu einer groben soziologischen Charakterisierung des heterotrophen Bewuchses herangezogen.

\section{Diskussion}

\subsection{Der zeitliche Verlauf der Biomassebildung in einem benthischen Berouchs}

Solange in einem benthischen Mikrophytenbewuchs bei konstantem Substratangebot die gesamte Schichtdicke $h$ hinlänglich versorgt wird, sind die Voraus- 
setzungen für ein exponentielles Wachstum gegeben. Der Zuwachs lässt sich dann durch folgende Beziehung umschreiben:

$B M=$ Biomasse

$$
\frac{d B M}{d t}=\mu B M=\mu \cdot F \cdot h \cdot X
$$

$\mu=$ Zuwachsrate

$C=$ Oberfläche des Bewuchses

$h=$ Schichtdicke

$X=$ Biomasse/Einheitsvolumen

Die Voraussetzungen für ein derartiges exponentielles Wachstum sind in den frühesten Stadien der Besiedlung eines Standorts gegeben und können z.B. in den Rinnen während der ersten 4-6 Tage nach der Exposition von Glasobjektträgern beobachtet werden (EICHENBERGER, unpubliziert). Mit zunehmender Dicke des Aufwuchses wird der Stoffaustausch mit den tiefer liegenden Zellschichten ungenügend. Die Dicke der noch aktiv bleibenden Schicht hängt vor allem von ihrer Durchlässigkeit für den Stofftransport ab (z.B. Geometrie der Poren, Anwesenheit von Organismen, die die Durchströmung fördern) und von der Stoffwechselaktivität der Zellen und der Substratkonzentration im Aussenmedium. Bei einem krustig ausgebildeten Aufwuchs wird diese Dicke 1. Millimeter kaum überschreiten $[16,11]$.

Hat ein Bewuchs einmal die maximal mögliche aktive Schichtdicke erreicht, so wird das Volumen der aktiven Biomasse von der Ausbildung seiner Oberfläche bestimmt. Damit kommt der Geometrie des Bewuchses, seiner räumlichen Anordnung im Biotop und der Struktur seiner Elemente eine grundlegende Bedeutung für den Verlauf des Biomassezuwachses zu.

Beim benthischen Aufwuchs können wir 2 Extremfälle der räumlichen Anordnung der Biomasse unterscheiden:

a) den schichtweisen Aufbau in Krusten,

b) die fädige Verteilung in der freien Wasserwelle.

Diese Unterschiede zwischen flächiger und zylindrischer Anordnung der Biomasse wirken sich zunächst auf die Dicke der versorgten Schicht aus [16]. Bei der krustenförmigen Ausbildung bleibt die Kontaktfläche zwischen Bewuchs und strömendem Medium über längere Zeit unverändert und damit auch das am Wachstum beteiligte Zellvolumen. Die Zuwachsgeschwindigkeit nimmt einen konstanten Wert an, und die Zunahme der Biomasse erfolgt dann proportional zur Zeit.

In fädigen und zottigen Bewuchsstrukturen hingegen vergrössert sich die dem Medium ausgesetzte Oberfläche laufend. Damit sollte der Wachstumsprozess beschleunigt ablaufen. Die Durchwachsung der benachbarten freien Wasserwelle verringert aber die Turbulenz und beeinflusst die Ausbildung der Grenzschichten [1] an der Bewuchsoberfläche, was zu ungünstigeren Austauschbedingungen führt. In Algenbiozönosen wird zusätzlich die zunehmende gegenseitige Beschattung der Fäden in steigendem Masse die Wachstumsgeschwindigkeit bremsen. Der angenähert zeitproportionale Zuwachs, der in den Rinnen bei zottigem Sphaerotilusbewuchs und in Algenfadenbiozönosen in der Besiedlungsphase beobachtet wird, weist darauf hin, dass die dem Wachstum förderliche Oberflächenzunahme weitgehend durch austauschvermindernde Strukturveränderungen des Bewuchses kompensiert wird. 


\subsection{Der Effekt der Abwasserkonzentration auf das Wachstum der Heterotrophen}

Wie aus dem Abschnitt 4.1 hervorgeht, lässt sich das benthische Wachstum wegen der Beschränkung der Austauschvorgänge im Bewuchs nicht durch eine Zuwachsrate $\mu$ charakterisieren. Damit stösst auch die Formulierung von Beziehungen zwischen Zuwachsgeschwindigkeit und Substratkonzentration unter Verwendung der für suspendierte Kulturen üblichen Parameter auf grundsätzliche Schwierigkeiten.

Kornegay und ANDrews [11] haben die von Monod abgeleiteten Beziehungen zwischen Wachstumsrate und Substratkonzentration auf biologische Filme in Reaktoren angewandt. Aus Messungen der Substratelimination bei bekannter aktiver Filmdicke erhielten sie einen scheinbaren $K_{s^{-}}$-Wert für Glukose von $121 \mathrm{mg} / \mathrm{l}$, einen Wert, der im Vergleich zu demjenigen von Mikroorganismen in Suspension recht hoch ausfällt.

Wenn wir davon ausgehen, dass die Zellen der Bewuchsoberfläche schon bei geringen Abwasserkonzentrationen ihre maximale Teilungsgeschwindigkeit erreicht haben, ist von einer weiteren Steigerung der Abwasserzufuhr keine Förderung ihres Wachstums zu erwarțen. Hingegen wird bei einer Erhöhung der Abwasserkonzentration einer zunehmend dickeren Schicht des Bewuchses das zum Wachstum nötige Substrat zugeführt; die Biomasseproduktion pro Flächeneinheit wird ansteigen. Die quantitative Beziehung zwischen Gesamtzuwachs und der Zunahme der Abwasserkonzentration ist das Ergebnis verschiedener physikalischer und biochemischer Eigenschaften des Biotops und des Bewuchses. In unseren Versuchen führt die Steigerung der $\mathrm{Abwasserkonzentration} \mathrm{bis} \mathrm{zur} \mathrm{maximalen} \mathrm{Beigabe} \mathrm{von} 12 \%$ Abwasser zu einer Biomasseproduktion (in g Glühverlust $/ \mathrm{m}^{2} \cdot \mathrm{Tag}$ ), die proportional zur Belastung ansteigt.

Bei einem Kohlenhydratgehalt (als org. C) des häuslichen Abwassers von etwa $30 \%$ des gelösten organischen Kohlenstoffs [13] ergibt sich für die höchste Abwasserkonzentration in unseren Versuchen ein mutmasslicher C-Gehalt der Kohlenhydrate von 2 bis $3 \mathrm{mg} / \mathrm{l}$ (etwa $30 \mu \mathrm{Mol}$ Monosaccharide/l). Lineare Zunahme des Zuwachses von Sphaerotilus natans bis zu $9 \mathrm{mg} / \mathrm{l}$ an löslichem C wurde in einem Rezirkulationskanal festgestellt [4], bei einer maximalen mittleren Zuwachsgeschwindigkeit von rund $1,5 \mathrm{~g}$ Trockensubstanz $/ \mathrm{m}^{2} \cdot$ Tag.

\subsection{Die jahreszeitlichen Veränderungen der heterotrophen Biomasseproduktion}

Die Wachstumsgeschwindigkeit der Heterotrophen bei der höchsten Abwasserbelastung von $12 \%$ variiert in Versuchen der Jahre 1967 und 1968 zwischen 2,3 und $6,2 \mathrm{~g}$ Glühverlust/Tag $\cdot \mathrm{m}^{2}$ (Abb. 5). Die Werte liegen zum Teil bedeutend höher als die $1,8 \mathrm{~g}$ Glühverlust $/ \mathrm{Tag} \cdot \mathrm{m}^{2}$, die in früheren Versuchen mit Melassezugabe von etwa $1,5 \mathrm{mg} \mathrm{C} / 1$ erreicht wurden (rund $330 \mathrm{~g} \mathrm{C}$ Tagesangebot) [19], und die Werte aus einer Laborrinne [4] von 1,2 $\mathrm{g}$ Glühverlust/Tag $\cdot \mathrm{m}^{2}$ auf Glukose und 1,8 $\mathrm{g}$ auf Acetat (bei etwa 7,6 g C Tagesangebot). Hingegen liegen sie in derselben Grössenordnung wie früher bestimmte Werte am gleichen Rinnensystem (Gesamtbiomasse in 10\% Abwasser von $\left.7 \mathrm{~g} / \mathrm{Tag} \cdot \mathrm{m}^{2}\right)[7]$.

Während die meisten Laboruntersuchungen bestes Wachstum von Sphaerotilus zwischen $25^{\circ}$ und $30^{\circ} \mathrm{C}$ feststellen, widersprechen sich die Angaben über die optimale Temperatur für die Ausbreitung im Freiland [3]. Die klimatische Situation in unseren 


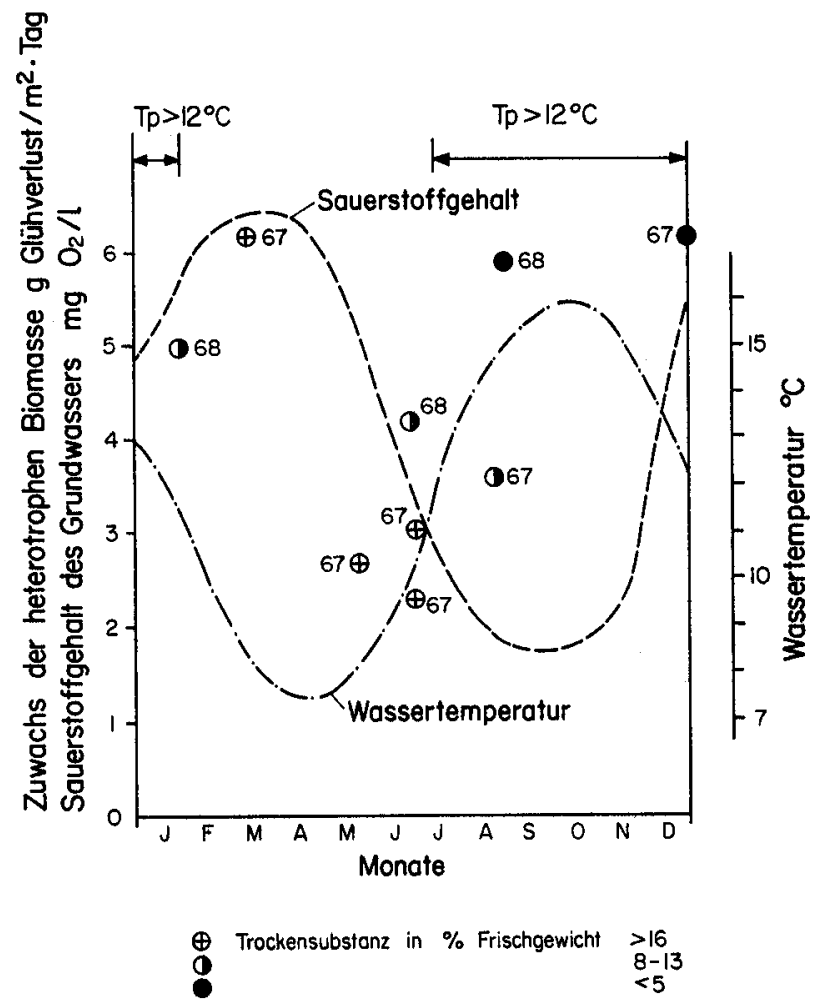

Abb. 5. Jahreszeitliche Verteilung der Zuwachsgeschwindigkeit des heterotrophen Anteils von Biozönosen in 12\% Abwasser.

Jeder Punkt ist das Mittel von 2 bis 4 Biomassebestimmungen im Laufe der Jahre 1967 und 1968. Probenahme am Anfang der Rinnen. Besiedlungsdauer: 14 bis 20 Tage.

Fig. 5. Seasonal distribution of the growth rates of the heterotrophic biomass in channels with $12 \%$ sewage.

Each point represents the average of 2 to 4 biomass determinations at the beginning of the channels in the years 1967 and 1968. Growth rate: $g$ organic substance (i.e. ignition loss)/day and $\mathrm{m}^{2}$ overgrown surface. Oxygen content and temperature: values for the groundwater flowing into the channels. These are representative of the conditons at the beginning of the channels. Sociological composition of the biocenosis: - low dry weight/fresh weight ratio with almost pure Sphaevotilus. $\oplus$ high dry weight/fresh weight ratio: fungal mycelia and fine filamentous bacteria dominate over Sphaerotilus.

Modellfliessgewässern weicht insofern von derjenigen natürlicher Gewässer ab, als zum Zeitpunkt der grössten Lichtintensität, im Frühsommer, die Wassertemperaturen tief liegen (unter $12^{\circ} \mathrm{C}$ ). Die vorliegenden Daten deuten auf eine Zunahme der Biomassebildung bei höheren Temperaturen hin, wobei eine Wechselwirkung mit der Lichtintensität besteht (Abb. 6).

Wie aus Beobachtungen an beschatteten Stellen und aus Abdunklungsversuchen hervorgeht, wird das Wachstum der Heterotrophen von der Belichtung beeinflusst (Tab. 2). In der Regel wird eine Hemmung durch das Licht festgestellt; in einem 
Falle hingegen wirkte die Belichtung fördernd. Auswirkungen des sichtbaren Lichts sind an verschiedenen Heterotrophen beobachtet worden [5, 2]. Die Effekte sind vielfältig, da das Licht verschiedene physiologische Vorgänge zu beeinflussen vermag

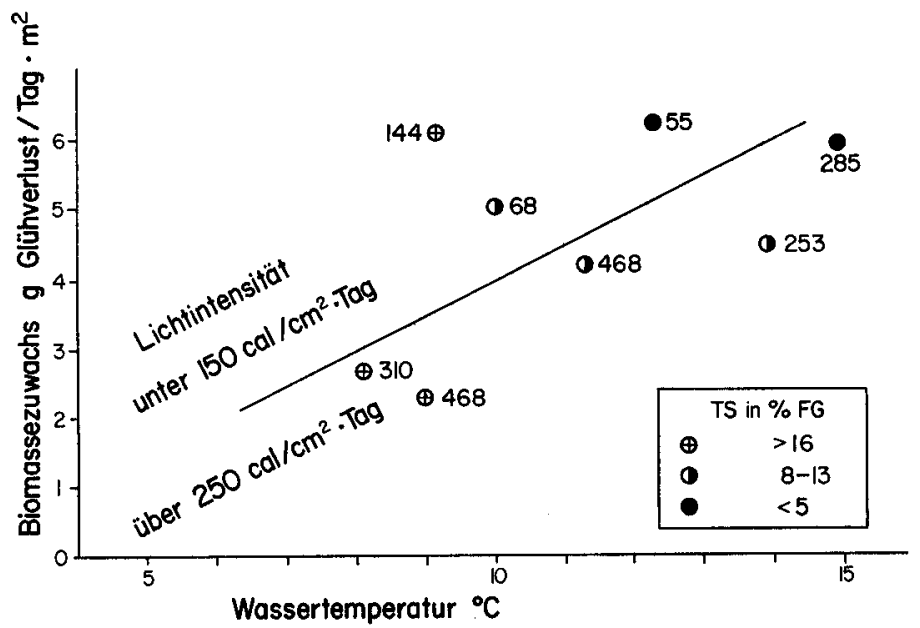

Abb. 6: Beziehung zwischen Wassertemperatur und Heterotrophenzuwachs bei $12 \%$ Abwasser. Jeder Punkt ist das Mittel von 2 bis 4 Biomassebestimmungen im Laufe der Jahre 1967 und 1968 am Anfang der Rinnen.

Messung der Lichtintensität: mit Kipp-Solarimeter und Integrator (Messbereich der Strahlung $2800 \AA-30000 \AA$ ). Besiedlungsdauer: 14 bis 20 Tage.

Fig. 6 Effect of water temperature on the heterotrophic growth rate in $12 \%$ sewage.

Each point represents the average of 2 to 4 biomass determinations at the beginning of the channels in the years 1967 and 1968 . Numbers: average light incidence in cal/day $\cdot \mathrm{cm}^{2}$, recorded with Kippsolarimeter and integrator (sensitivity range from $2,800 \AA$ to $30,000 \AA$ ). Growth rate: g organic substance (i.e. ignition loss)/day and $\mathrm{m}^{2}$ overgrown surface. Duration of colonisation: 14 to 20 days.

Tabelle 2. Effekt des Lichtes auf das Wachstum der heterotrophen Komponente der Biozönosen.

\begin{tabular}{|c|c|c|c|c|c|c|c|c|}
\hline \multirow{3}{*}{ Datum } & \multirow{3}{*}{$\begin{array}{l}\text { Wasser- } \\
\text { temperatur } \\
{ }^{\circ} \mathrm{C}\end{array}$} & \multirow{3}{*}{$\begin{array}{l}\text { Licht } \\
\mathrm{cal} / \mathrm{cm}^{2} \cdot \mathrm{Tag}\end{array}$} & \multirow{3}{*}{$\begin{array}{l}\mathrm{BSB}_{5} \\
\mathrm{mg} \mathrm{O}_{2} / \mathrm{I}\end{array}$} & \multicolumn{5}{|c|}{ Zuwachsgeschwindigkeit } \\
\hline & & & & \multicolumn{3}{|c|}{$\begin{array}{l}\text { phototropher hetero } \\
\text { Komponenten } \\
\text { g Gluhhverlust/Tag } \cdot \mathrm{m}^{2}\end{array}$} & \multirow[b]{2}{*}{ Dunkel } & \multirow[t]{2}{*}{$p$} \\
\hline & & & & Licht & Dunkel & Licht & & \\
\hline Mai 1967 & 7,8 & 310 & 19,2 & 0,53 & 0,07 & 2,70 & 3,80 & $<0,01$ \\
\hline Juni 1967 & 9,0 & 467 & 21,4 & 0,39 & 0,06 & 3,44 & 5,35 & $0,1-0,05$ \\
\hline August 1967 & 14,0 & 253 & 31,0 & 2,77 & 0,11 & 2,72 & 5,42 & $<0,01$ \\
\hline August 1968 & 15,0 & 285 & 13,1 & 2,15 & 0,12 & 4,26 & 3,09 & $<0,05$ \\
\hline
\end{tabular}

Lichtintensitäten: Mittelwerte der Jahre 1970/71 (gemessen mit Kipp-Solarimeter); $\mathrm{BSB}_{5}$ des Rinnenwassers bei 12\% Abwassergehalt; $p$ Wahrscheinlichleit für Zutreffen der Nullhypothese nach t-Test; Messungen der Biomasse nach einer Fließstrecke von 20 bis $30 \mathrm{~m}$. 
und verschiedene Wirkungen sich überlagern können. Die Natur der Lichtbeeinflussung heterotropher Mischbiozönosen unter natürlicher Belichtung bleibt vorderhand offen. In Sphaerotilusreinkulturen, die in die Rinnen eingetaucht waren und damit unter Freilandklima standen, sind sowohl Hemmung wie Förderung des Wachstums beobachtet worden (MECHSNER, unpubliziert). In natürlichen Biozönosen ist damit zu rechnen, dass neben der direkten Einwirkung von Belichtung und Temperatur auf die Physiologie der Heterotrophen auch indirekte Lichteffekte auftreten, die auf Veränderungen in der Soziologie der Lebensgemeinschaft zurückzuführen sind.

Daten für die Intensität des Lichteinfalls stehen für die Jahre, in denen die Versuche durchgeführt wurden, nicht zur Verfügung. Da über eine Versuchsperiode von 14 bis 20 Tagen die Unterschiede des Lichteinfalls zwischen verschiedenen Jahren relativ klein sind, wurden die Werte der Jahre 1970/71 als Vergleichsbasis herangezogen. Bei der geringen Tiefe des Wassers (maximal $15 \mathrm{~cm}$ ) und der geringen Trübung wird die Lichtintensität in den Kanälen nur wenig reduziert und liegt im Laufe des Jahres zwischen etwa 50 und $450 \mathrm{cal} / \mathrm{cm}^{2}$. Tag (gemessen mit einem Kipp-Solarimeter). Das Heterotrophenwachstum fällt bei tiefen Wassertemperaturen mit steigendem

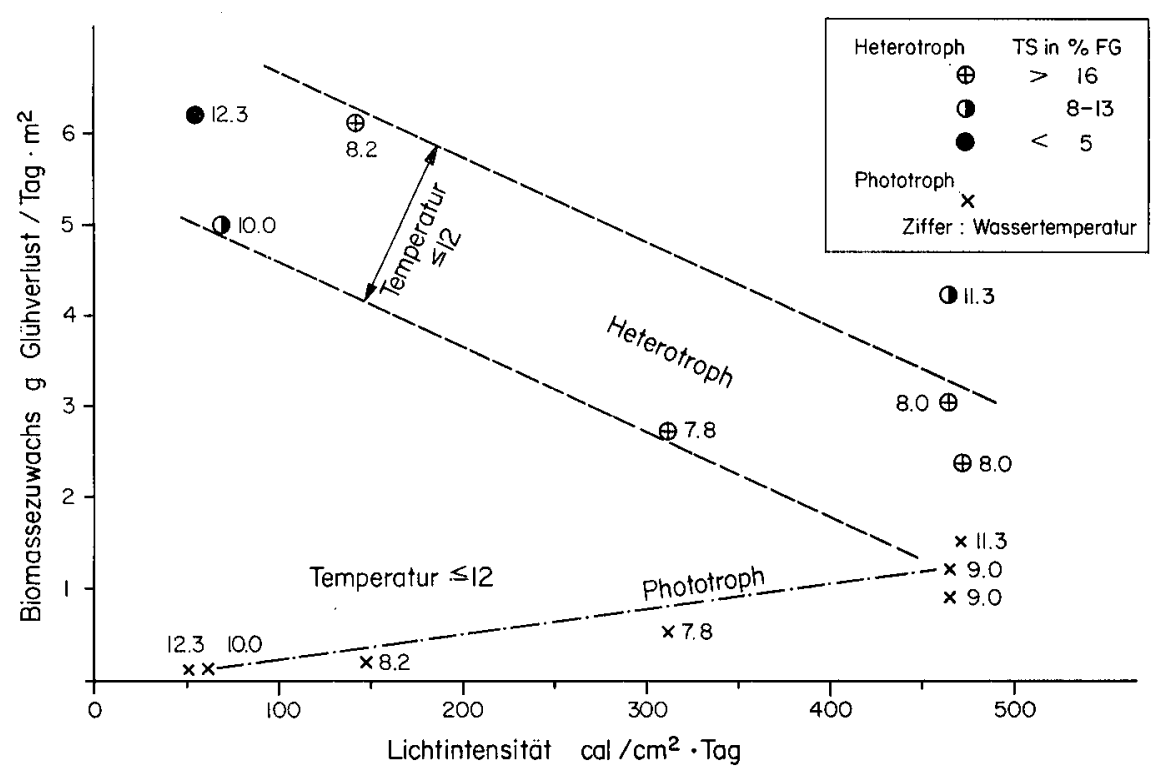

Abb. 7. Wirkung der Lichtintensität auf heterotrophen und phototrophen Zuwachs bei $12 \%$ Abwasser.

Jeder Punkt ist das Mittel von 2 bis 4 Biomassebestimmungen im Laufe der Jahre 1967 und 1968 am Anfang der Rinnen. Einzig Versuche bei mittleren Wassertemperaturen um $12^{\circ} \mathrm{C}$ wurden berücksichtigt. Besiedlungsdauer: 14 bis 20 Tage.

Fig. 7. Effect of light incidence on heterotrophic and phototrophic growth rates in $12 \%$ sewage at low water temperatures.

Each point represents the average of 2 to 4 biomass determinations at the beginning of the channels in the years 1967 and 1968. Numbers: water temperatures. Growth rate: $g$ organic substance

(i.e. ignition loss)/day and $\mathrm{m}^{2}$ overgrown surface. Duration of colonisation: 14 to 20 days. 
Lichteinfall deutlich ab (Abb. 7). Bei Temperaturen unter $10^{\circ} \mathrm{C}$ und bei $12 \%$ Abwasserzusatz nimmt das Phototrophenwachstum mit steigender Lichtintensität zu. Zwischen der Biomasse der Heterotrophen und der Phototrophen besteht unter diesen Bedingungen eine negative Korrelation.

CURTIS [3] hat festgestellt, dass die Kolonisation durch Sphaerotilus bei einem Sauerstoffgehalt des Wassers von über 5-6 mg/l erschwert ist. In unseren Anlagen sind Temperatur und Sauerstoffgehalt des Wassers unabhängige Variablen und bewegen sich mehr oder weniger gegenläufig. Im vorliegenden Konzentrationsbereich von 2 bis $6 \mathrm{mg} \mathrm{O/l}$ am Rinnenanfang lässt sich keine Auswirkung auf die Soziologie des heterotrophen Komponenten des Bewuchses und ihr Wachstum feststellen (Abb. 5).

Die Konzentration des den Rinnen zudosierten Abwassers ist sehr raschen und grossen Schwankungen unterworfen, ohne dass aber ein deutlicher jahreszeitlicher Rhythmus im $\mathrm{BSB}_{5}$ nachweisbar ist [6]. Der Heterotrophenzuwachs verschiedener Versuchsperioden zeigt keine Korrelation zum $\mathrm{BSB}_{5}$ des $\mathrm{Abwassers} \mathrm{(Abb.8),} \mathrm{obschon} \mathrm{für} \mathrm{einen}$
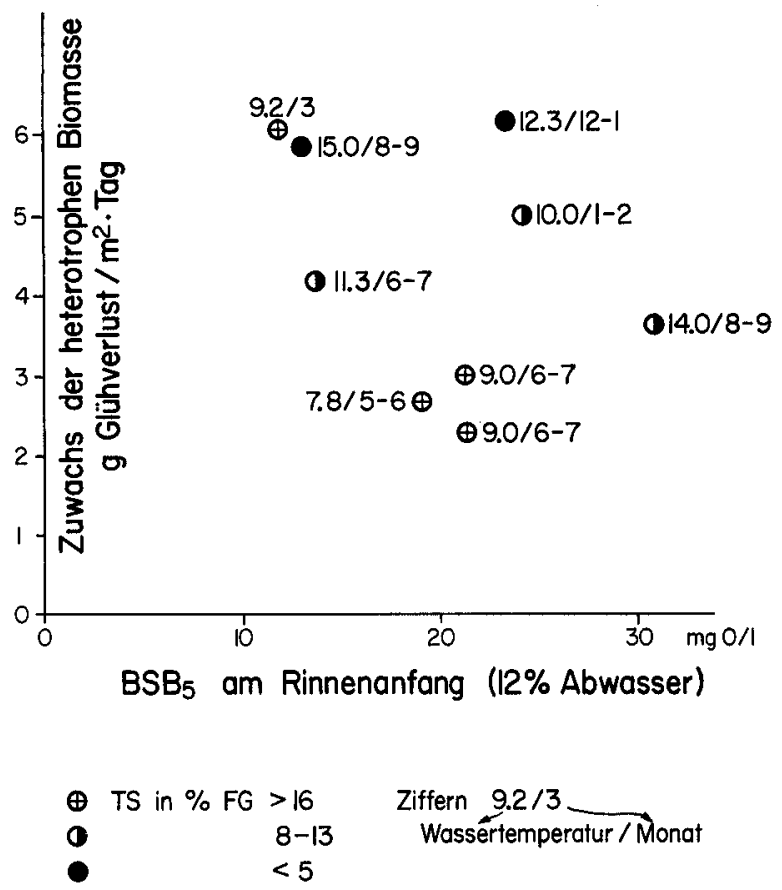

Abb. 8. Beziehung zwischen Heterotrophenzuwachs und $\mathrm{BSB}_{5}$ bei $12 \%$ Abwasser.

Jeder Punkt ist das Mittel von 2 bis 4 Biomassebestimmungen im Laufe der Jahre 1967 und 1968

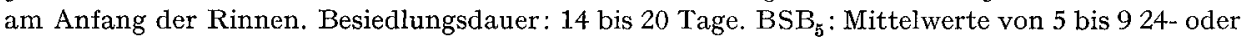
72 stündigen Sammelproben, gemessen bei $20^{\circ} \mathrm{C}$ im Dunkeln. Besiedlungsdauer: 14 bis 20 Tage.

Fig. 8. Dependence of the heterotrophic growth rate in $12 \%$ sewage on the BOD of the water. Each point represents the average of 2 to 4 biomass determinations at the beginning of the channels in the years 1967 and 1968. First number: average water temperature, second number: month. $\operatorname{BOD}_{(5 \text { days) }}$ : average at the beginning of the channels. 
einzelnen Versuchsabschnitt Proportionalität zwischen Abwasserbelastung und Wachstum nachgewiesen wurde. Temperatur und besonders Lichtintensität sind unter den vorliegenden Bedingungen die bedeutungsvolleren Variablen als die mit dem $B S B_{5}$ gemessene Konzentration des Abwassers.

Die bei den Abdunklungsversuchen festgestellte Steigerung der Bewuchsbildung beruht möglicherweise darauf, dass unter natïrlichen Belichtungsbedingungen der Abbau gewisser Komponenten des Abwassers verzögert ist oder anders verläuft als bei der Ermittlung des $\mathrm{BSB}_{5}$ im Dunkelraum, so dass schon aus diesem Grunde eine Korrelation zum BSB $_{5}$ nicht zu erwarten ist. Damit drängt sich der Schluss auf, dass eine erfolgversprechende Analyse der Wirkung von Abwasser verschiedener Zusammensetzung auf die Entwicklung der Heterotrophen unter natürlichen Bedingungen mehr analytische Angaben erfordert, als der $\mathrm{BSB}_{5}$ sie hergibt.

In einem Fliesswassermodell, das mit Grundwasser gespiesen wird und dessen Temperatur eine Phasenverschiebung gegenüber den benachbarten Oberflächengewässern von etwa 3 Monaten aufweist, kommt der Beimpfung mit vermehrungsfähigen Keimen eine entscheidende Bedeutung für die Ausbildung des Algenbewuchses zu. Sofern nicht eine rinneneigene Beimpfung aus einem stabilisierten Abschnitt erfolgen kann, besteht die Möglichkeit, dass die aus der Umgebung eingeführten Keime im andersartigen Temperaturbereich der Rinnen ungünstige Vermehrungsbedingungen vorfinden. Die ausgesprochen schlechte Entwicklung derjenigen Fadenalgen, die in früheren Versuchen die Biomasse beherrschten [6], lässt sich wohl teilweise auf die ungünstigen Beimpfungsverhältnisse zurückführen, nachdem die Rinnen vor jedem Versuch völlig gereinigt wurden. Wir dürfen deshalb annehmen, dass unter günstigeren Beimpfungsbedingungen die Veränderungen im jahreszeitlichen Verhältnis zwischen Phototrophen und Heterotrophen noch ausgeprägter ausfallen würden.

\section{Zusammenfassung}

1. In Freilandfliessgewässermodellen wurde die jahreszeitliche Veränderung der Biomassebildung von benthischen Mikrophyten bei $0,1,5$ und $12 \%$ Abwasserzugabe während einer Besiedlungsdauer von 20 Tagen untersucht.

2. Die heterotrophe Biomasse nimmt in $12 \%$ Abwasser während der ersten 3-4 Wochen der Besiedlung freier Flächen linear zur Besiedlungszeit zu und ist für einzelne Versuchsabschnitte zwischen 0 und $12 \%$ Abwasserzugabe proportional der Abwasserkonzentration.

3. Das Verhältnis von phototrophen zu heterotrophen Mikrophyten verändert sich bei gleichbleibender Abwasserbeimischung jahreszeitlich. Beste Ausbildung der Phototrophen erfolgt im Sommerhalbjahr, wenn die Bedingungen für die Heterotrophen weniger günstig sind als im Winter. Bei 5\% Abwasser machen die Phototrophen im Winter etwa $10 \%$, im Sommer bis zu $70 \%$ der Gesamtbiomasse aus, bei $12 \% \mathrm{Ab}$ wasser liegt ihr entsprechender Anteil bei 2 bzw. 30\%.

4. Die Belichtungsbedingungen sind eine wichtige Variable für das Wachstum der heterotrophen Mikrophyten. 


\section{SUMMARY}

1. The seasonal changes of the biomass production by benthic microphytes have been investigated in outdoor channels (breadth $25 \mathrm{~cm}$, depth $10-20 \mathrm{~cm}$, flow $4 \mathrm{l} / \mathrm{sec}$ ). The channels were fed with groundwater to which $0,1,5$ and $12 \%$ settled domestic sewage was added as pollutant.

2. In the course of the colonisation of freshly exposed surfaces the biomass increases approximately linear with time for at least 3 weeks. Later on, depending on the organisms present, a more or less marked spontaneous detachment of the growth occurs (Fig. 1 and Fig. 2). The investigation of the effect of sewage on the production of biomass was therefore limited to the first 20 days of colonisation, so that an average daily rate of biomass increase could be computed (defined as $g$ organic biomass - i.e. ignition loss-per day and square meter overgrown surface).

3. The ratio between phototrophs and heterotrophs is expressed as $\mathrm{P} / \mathrm{H}$ index (i. e. the ratio between the chlorophyll content and the biomass of the biocenosis). For a pure phototrophic biocenosis this $\mathrm{P} / \mathrm{H}$ index is around 40 units, for pure heterotrophic ones around 1 unit. By assuming that in all the biocenosis the phototrophic growth has a $\mathrm{P} / \mathrm{H}$ index of 40 units, the biomass of the phototrophs and the heterotrophs can be estimated separately (page 157).

4. The comparison between different channels run under the same conditions points to a good reproducibility of the biomass production (Fig. 2 and 3 ).

5. The heterotrophic biocenosis varies in its sociological composition. Dense, matlike growth with a high proportion of fungal mycelia, fine filamentous bacteria (probably flavobacteria) and relatively few Sphaerotilus, with a high dry weight to fresh weight ratio are more common at low water temperatures. The typical long tufted, Sphaerotilus dominated biocenosis with a low dry weight to fresh weight ratio predominates when the water is warmer (Fig. 6).

Although the blooms of Ulothrichaceae in spring and of Cladophora in summer which were typical for the vegetation of the channels in earlier years (EICHENBERGER 1967 a) have not occurred, there is a clear seasonal variation in the sociology of the phototrophs (Table 1).

6. For each season tested the heterotrophic growth is proportional to the sewage concentration within the range from 0 to $12 \%$. The growth is faster in winter (about $6 \mathrm{~g}$ organic biomass/ $\mathrm{m}^{2}$. day) than in summer. Optimal conditions for the phototrophs occur in summer and lie between 1 and $5 \%$ sewage (Fig. 4). As a consequence a yearly periodicity of the ratio between phototrophic and heterotrophs is observed (Fig. 4).

7. No correlation was noticed between the growth rate of the heterotrophs and the BOD of the water in a number of experiments with $12 \%$ sewage (Fig. 8). However light incidence appears to be an important variable for the growth of heterotrophs (Fig. 7).

Die vorliegende Arbeit ist in entscheidender Weise durch die Anregungen von Herrn Prof. Dr. K. Wuhrmann gefördert worden, und ich möchte ihm für seine Unterstützung bei der Ausführung und Bearbeitung der Untersuchungen herzlich danken. An unser technisches Personal (vor allem die Herren H. Schälchli, J. Stäheli und E. Höhn) geht meine dankbare Anerkennung für die zuverlässige Wartung der Versuchsanlage.

\section{LITERATURVERZEICHNIS}

[1] АмвÜнц, H., Die Bedeutung der Strömung als ökologischer Faktor, Schweiz. Z. Hydrol, 21, 133-263 (1962).

[2] Carlile, M. J., The Photoresponses of Fungi, in: Photobiology of Microorganisms, S. 39-344 (Ed. Per Halldal, Wiley, New York 1970).

[3] Curtis, E. J. C., Sewage Fungus: its Nature and Effects, Water Research 3, 289-311 (Pergamon Press, London 1969).

[4] Curtis, E. J. C., Delves-Broughton, J., und Harrington, D. W., Sewage Fungus: Studies of Sphaerotilus Slimes using Laboratory Recirculation Channels, Water Research 5, 267-279 (Pergamon Press, London 1971).

[5] EHREnBerg, M., Die Höhe des Lichteffektes auf Wachstum und Stoffwechsel von Sacchavomyces cerevisiae in Abhängigheit vom Phasenstatus der Vorkulturzellen, Arch. Mikrobiol. 55, 20-29 (1968). 
[6] EICHENBERGER, E., Öhologische Untersuchungen an Modellfliessgewässern. I. Die jahreszeitliche Verteilung der bestandesbildenden pflanzlichen Organismen bei verschiedener Abwasserbelastung, Schweiz. Z. Hydrol. 29, 1-31 (1967).

[7] Eichenberger, E., Ökologische Untersuchungen an Modellfliessgewässern. II. Jahreszeitliche Veränderungen der Biomassebildung bei verschiedenen Abwasserbelastungen, Schweiz. $Z$. Hydrol. 29, 32-52 (1967).

[8] EICHENBERGER, E., Ökologische Untersuchungen an Modellfliessgewässern. IV. Auswirkung der Selbstreinigung auf die Biomassebildung in einem Abwassergradienten, Schweiz. Z. Hydrol. 34, 173-189 (1972b).

[9] Eichenberger, E., und WuHrmanN, K., Jahreszeitliche Veränderungen des Pflanzenbezenchses in Modellfliessgewässern mit verschiedener Abwasserbelastung, Verh. int. Verein. theor, angew. Limnol. 16, 888-896 (1966).

[10] Mechsner, K., Das Wachstum von Sphaerotilusveinkultuven in vitro unter natiulichen Klimabedingungen, Interner Bericht EAWAG (1968, unpubliziert).

[11] Kornegay, B. H., und Andrews, J. F., Kinetics of Fixed-film Biological Reactors. J. Wa1. Poll. Contr. Fed. 40, 460-468 (1968).

[12] Phaup, J. D., und Gannon, J., Ecology of Sphaerotilus in an Experimental Outdoor Channel, Water Research 1, 523-541 (1967).

[13] Painter, H. A., und Viney, M., Composition of Domestic Sewage, J. biochem. microbiol. Technol. Eng. 1, 143-162 (1959).

14] Rabinowitch, E. I., Photosynthesis and Related Processes, Vol. II/1, 603, 1208 (Interscience, New York 1951).

[15] Wuhrmann K., Über die biologische Prüfung von Abwasserreinigungsanlagen, GesundheitsIngenieur 72, 1-9 (1951).

[16] Wuhrmann, K., Effect of Oxygen Tension on Biochemical Reactions in Sewage Purification Plants, in: Adv. Biol. Waste Treatm. (Ed. Eckenfelder \& Mc Cabe, Pergamon Press, New York 1963).

[17] WuHrmann, K., River Bacteriology and the Role of Bacteria in Self-purification of Rivers, in: Principles and Application in Aquatic Microbiology, S. 167-192 (Ed. Heukelekian and Dondero Wiley, New York 1964).

[18] Wuhrmann, K., Self Purification, in: Microbiology of Polluted Waters (Ed. R. Mitchell, J. Wiley, New York 1972).

[19] Wuhrmann, K., Ruchir, J., und Eichenberger, E., Quantitative Experiments on Selfpurification with Pure Organic Compounds, Third Int. Conf. Wat. Poll. Res. (1969).

Anschrift des Autors:

Elie Eichenberger, Dr.rer. nat., EAWAG, Versuchsanlage "Tüffenwies», CH-8048 Zürich, Schweiz. 